\title{
LA EDUCACIÓN MATEMATICA: UNA DISCIPLINA EN FORMACIÓN
}

\author{
CARLOS E. VASCO U. \\ Universidad Nacional de Colombia
}

1. LAS

MATEMATICAS COMO PROCESO

En el presente artículo me propongo precisar lo que podemos entender por "Educación matemática" como una nueva disciplina en proceso de conformación, y ubicarla en relación con las matemáticas y con otras disciplinas pertinentes

Empecemos por las matemáticas. Lo que se entiende por "Matemáticas" no es tan claro como parece a primera vista. No hay propiamente una definición de las matemáticas, y las decenas de definiciones o descripciones propuestas no aglutinan más que un pequeño grupo de seguidores. Pero no puede dudarse de la existencia en la historia de procesos sociales de ideación, discusión, consignación en

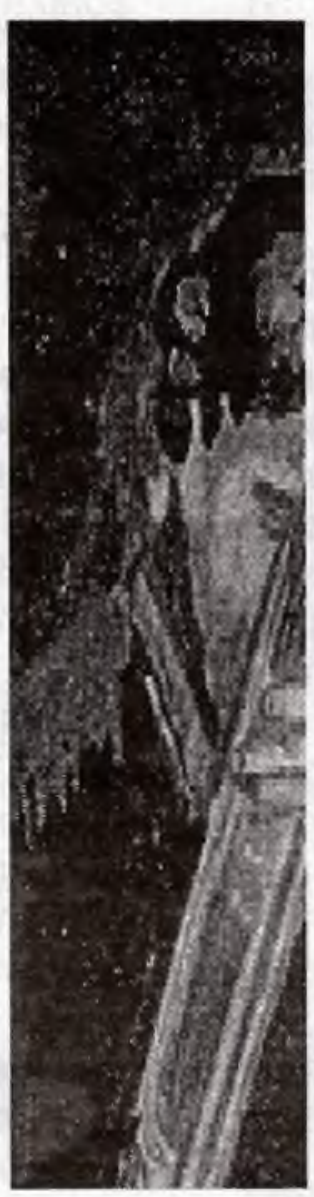

símbolos y gráficas, decodificación y refundición de saberes, que por lo menos quienes lo practican identifican claramente como matemáticas.

Por razones analíticas podemos distinguir unos procesos culturales que podríamos llamar las "matemáticas realmente existentes", que incluyen el conteo de personas $y$ animales, los cumpleaños y los ciclos solares y lunares, los días, las horas, el dinero y el comercio, los inventarios y avalúos, las finanzas, las estadísticas, los ábacos, las calculadoras y las computadoras, los números de teléfonos, las direcciones, las temperaturas, etc. Tendríamos pues la matemáticas realmente existentes como procesos que tienen existencia social independiente de la educación matemática y de la investigación matemática de punta. 
Pero en esos mismos grupos sociales en donde fluyen las matemáiticas realmente existentes, llega el momento en que se van creando ciertos dispositivos que podriamos llamar "la pedagogía de las matemáticas", surgen personas que las enseñan a otras personas, así sea de manera informal. Hay quienes les enseñan a otros a contar, la manera de escribir y manejar cierto vocabulario, ciertas acciones y símbolos, etc. Esa es la pedagogía de las matemáticas como práctica social. No estoy pensando en la disciplina moderna llamada "pedagogía o didáctica de las matemáticas", sino en la práctica pedagógica de comunicar matemáticas.

Al constítuirse esos dispositivos pedagógicos, se van concentrando unas capas superiores más refinadas, que van adquiriendo cada vez más independencia, hasta constituír la investigación en matemáticas, o las matemáticas de punta, 0 las matemáticas puras.

Tendriamos pues al menos tres ramales de una trenza diacrónica: las matemáticas realmente existentes en la cultura, la pedagogía de las matemáticas o matemáticas escolares, y las matemáticas de investigación. Insisto en que la diferencia entre esos tres ramales es más bien teórica y analítica; muchas veces somos las mismas personas las que sabemos contar, las que tratamos de cuadrar la chequera y nos equivocamos en las sumas, las que luego pasamos a enseñar matemáticas, o nos dedicamos a la investigación matemática más refinada. Por lo tanto, también podríamos decir que las matemáticas de investigación y las escolares son matemáticas realmente existentes. En la cultura todos estos procesos son cíclicos y se realimentan. La persona que aprende a contar antes de que se lo enseñen en pre-escolar, que empieza a aprender a manejar por si misma las matemática realmente existentes en su cultura puede convertirse en un buen pedagogo de las matemáticas, $y$ si cuenta con el apoyo de otros buenos pedagogos puede llegar también a ser un productor de matemáticas de nivel investigativo. Esas matemáticas de punta pueden volver a alimentar las matemáticas de la vida cotidiana, que es la que está continuamente alimentando, criticando, dando nuevas, oportunidades de refinamiento y desarrollo a las matemáticas en cualquiera de sus formas. Una vez completado el ciclo, aparecen nuevas matemáticas de investigación cada vez mis refinadas, que entre otras cosas, parecen afectar cada vez menos la vida cotidiana, si no es indirectamente a través de las tecnologías.

Tratemos pues de pensar este proceso social de las matemáticas como si fuera fluyendo, caminando en el tiempo, y distinguiendo en el las matemáticas realmente existentes, las matemáticas escolares como prácticas pedagógicas de enseñanza de las matemáticas, y las matemáticas de investigación.

Respecto a estos procesos, podemos adoptar dos posiciones o miradas básicas: la de dentro y la de fuera. La mirada de dentro es la mirada del practicante, del que está inmerso en una práctica. Por ejemplo, si las 
matemáticas realmente existentes en las que nos estamos fijando estan representadas por la contabilidad de una empresa, es posible que la persona que lleva esa contabilidad emplee una serie de algoritmos rápidos que no aparecen en los libros usuales, por ejemplo algunos de esos trucos que aparecen en el método Trachtenberg, que ninguna persona normal sigue, pero que tienen efectos sorprendentes en rápidez y eficiencia. Ese mismo contador en sus ratos libres puede convertirse en un Fermat $y$ desarrollar abstrusos teoremas, o puede simplemente repetir sus prácticas cotidianas de contador sin pensarlas siquiera, o puede enseñarlas a un aprendiz sin pensar que está pasando de practicante a pedagogo de las matemáticas. Puede por ejemplo utilizar el conocido algoritmo para multiplicar por once un número de dos cifras: la suma, y escribe ese total entre las dos cifras originales. Demostrar que eso es válido es muy díficil, y generalizarlo a tres o más cifras es más díficil todavía. Sin embargo, a la mayoría de las personas les llama mucho la atención ese algoritmo; pero muy pocos se preguntan siquiera por qué funciona, o si funciona siempre o no. Pero en un momento dado, esa persona puede empezar a explorar el algoritmo mismo, a demostrar que siempre funciona, a extenderlo a un mayor número de cifras, etc. El practicante puede pues actuar en cualquiera de los tres ramales del proceso con mayor o menor conciencia de lo que se hace.

Esa visión de adentro ocurre también en las matemáticas escolares, o sea en lo que hemos llamado "la pedagogía de las matemáticas". Es la visión del maestro que sabe matemáticas al nivel que él considera necesita saberlas. Por ejemplo enseña cálculo en grado once, y se ofende cuando un profesor universitario dice que no se puede enseñar cálculo sin haber tomado cursos de análisis complejo; en realidad, el no ha tomado esos cursos, pero si sabe que el análisis complejo no es necesario para enseñar bien el cálculo, y con razón se siente atropellado por los matemáticos de investigación. Ese profesor que no sabe análisis complejo si puede saber mucho sobre su vida cotidiana como maestro de matemáticas, sobre la de sus alumnos y la manera como ellos aprenden los conceptos de límite, continuidad, derivada, integral indefinida y definida; sobre las dificultades que tienen con la interpretación de las gráficas, sobre las maneras como se enseñan unos a otros, etc. Ese saber pedagógico del maestro de matemáticas puede tener limitaciones, como por ejemplo creer que los alumnos aprenden lo que uno les enseña, sin caer en la cuenta de que el proceso de enseñanza está muy lejos del de aprendizaje, hasta el punto de que con frecuencia llegan a ser contradictorios. Pero ese saber que él tiene sobre los procesos que el vive cuando enseña, cuando evalúa, cuando acompaña a sus alumnos en el aprendizaje, es muy importante como saber pedagógico, como saber sobre la educación matemática desde dentro del proceso.

No se trata de crear distinciones inútiles entre los matemáticos de investigación, los educadores matemáticos y los practicantes cotidianos de las matemáticas, sino de ponerlos 


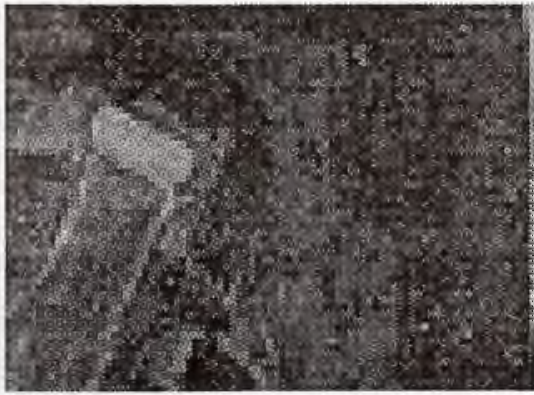

en red, de reunir a todos esos practicantes de las matemáticas realmente existentes, de las escolares y de las de investigación, para que por lo menos un buen número de ellos adopten también la mirada desde fuera.

Pero si parece que algunos de nosotros tenemos una vocación, asi $\Re$ considere una especie de deformación mental, y es que nos gusta mirar los procesos desde fuera. Esa posición no sale gratis: tiene sus costos. Pensemos en una persona que va a una fiesta a observar, a mirar qué hace la gente, etc. Puede resultarle interesante asumir esa posición de antropólogo, pero el costo que paga es que se pierde de lo bueno de la fiesta, y hasta se convierte en un aguafiestas. Puede observar detalles muy interesantes respecto a la manera como la gente llega, saluda, forma grupos, excluye consciente o inconscientemente a otros, etc. Puede que hasta logre publicaciónes muy finas y profundas sobre la microsociología de las fiestas. Pero el que se dedicó a registrar esos aspectos, probablemente no disfrutó del baile, del licor, del buen ambiente, porque sencillamente estaba en otro plan. Tenemos pues que apreciar, o al menos tolerar, a los que miran desde fuera los procesos. Y para precisar lo que es esa nueva disciplina de la educación matemática, y para poder ubicarla con respecto a otras disciplinas, es necesario tomar esa posición externa y adoptar esa mirada desde fuera.

\section{EL OCTOGONO DE LA EDUCACION MATEMATICA}

Tratemos pues de situar la educación matemática como disciplina, visualizándola desde una posición externa, con una mirada desde fuera de ese proceso triple de las matemáticas realmente existentes, las escolares y las de investigación. Esa nueva disciplina se ubica a su vez dentro de un octógono de disciplinas que permiten pensarla como distinta de ellas, pero a su vez como impensable sin ellas. Este octógono que hoy propongo tuvo su origen en el hexágono de la ciencia cognitiva que presenta Howard Gardner en su libro "La nueva Ciencia de la Mente", adaptado a la educación matemática.

Las ocho disciplinas que conforman el octógono de la educación matemática no representan saberes en los cuales un practicante de la educación matemática debería ser experto o especialista; pero sí representan disciplinas sobre las que el practicante de la educación matemática, si quiere convertirse en investigador en educación matemática, debe tener suficiente información para poder calificar su mirada desde fuera y su mirada desde dentro de los procesos relacionados con la educación 
matemática. Por supuesto que es improcedente exigirle a una persona que está enseñando en dos jornadas de secundaria, $y$ tal vez estudiando en la noche en una universidad, que se proponga sacar ocho Ph.D. antes de empezar a investigar, en educación matemática, sin contar otro Ph.D. en matemáticas y uno más en educación. Pero sí se le puede pedir que sea consciente de que va a tener encuentros, iluminaciones y conflictos con esas ocho disciplinas, que va a encontrar traslapes 0 intersecciones en ellas, y que todo lo que aprenda, de ellas va a darle aportes muy básicos para la práctica cualificada y sería de la investigación en educación matemática. Veamos Cuáles son esas ocho disciplinas del octógono.

La más básica de todas es la biología, en particular la neurología. Ubiquémosla en el vértice inferior, en la base de ese octógono. En forma paralela a lo que ocurre con la ciencia cognitiva, estamos todavía lejos del día en que sepamos tanto sobre el funcionamiento del cerebro, de los hemisferios, las circunvoluciones y las neuronas, que ya podamos derivar de ese conocimiento la forma como podemos tratar un problema lógico, o sepamos cómo se almacena cada tipo de información, o en que región del cerebro se almacenan distintos tipos de algoritmos. Pero la neurología sigue empujando las fronteras del saber de abajo hacia arriba, y en el futuro será cada vez más pertinente a la investigación en educación matemática.

Vamos ahora al otro extremo del octógono. La filosofía está influyendo cada vez más en la investigación en educación matemática, así sea de manera implícita. Podríamos hablar de la filosofía implícita del practicante de las matemáticas de investigación, o del educador matemático. Entre los aspectos filosóficos de la ciencia congitiva se consideraba sólo la epistemología. Pero la epistemología es un poco restrictiva, en el sentido de que trata de los saberes que se llaman a sí mismos científicos. Habría que considerar una región mas amplia, ese tratado filosófico sobre el conocimiento que se llamaría gnoseología o teoría del conocimiento en general, del cual el conocimiento científico no es más que una región más estrecha. Se ha visto por ejemplo que el conocimiento no científico de los practicantes es mucho mas importante que lo que pensaban los científicos e investigadores.

Pero además de ese tratado filosófico sobre el conocimiento, la filosofía tiene mucho que aportar a la educación matemática. Pronto aparecen preguntas sobre el por qué y el para qué de aprender matemáticas; sobre qué contribuciones hacen ellas a la cultura, etc. Esas preguntas exigen una visión más amplia de la persona humana y de la sociedad, que sólo podría darla una antropología filosófica, que no puede restringirse ni a la metafísica ni a la gnoseología. Hay que reflexionar sobre aspectos como la sensibilidad y la estética, y cada vez se ve más claro que hay que reflexionar sobre la ética. Esa antropología filosófica nos orientaría sobre lo que es y debe, ser la sociedad, el hombre, el comportamiento ético, el papel de lo estético, etc. La teoría de la acción comunicativa de Habermas trata de recalcar lo razonable (no digamos lo racional, ni 
menos lo racionalista) de dialogar sobre aspectos de la ética, la estética, el hombre y la sociedad, etc., y no restringir la racionalidad a la lógica 0 a lo meramente objetivo y científico. Ubiquemos pues la filosofía con todas las ramas mencionadas en el vértice superior del octógono.

Habría quienes ubican la lógica como otro tratado filosófico, pero prefiero ponerla como disciplina específica en un vértice cercano del octógono, arriba a la derecha, ya sea que se le considere como parte de la filosofía, o como parte de las matemáticas, 0 como disciplina metalingüística.

La lingüística misma, o mejor todavía, una semiología o semiótica general, debería ocupar otro vértice del octógono, por ejemplo el vértice central derecho. Es imposible investigar sobre cómo se enseña o se aprende a contar sin caer en la cuenta de finuras lingüísticas como el cambio que hay entre el quince y el dieciseis. La palabra "quince" no tiene referencia al diez, así la tenga en el latín "quindecim". Pero "dieciseis" ya tiene una clara alusión al diez y al seis. No podemos tampoco dejar pasar desapercibidos los pequeños cambios lingüísticos que ocurren con las palabras "veinte", "treinta", etc. Las palabras de veinte a noventa tienen una estructura lingüística diferente al de las palabras del doscientos al novecientos. Si se le fomentara al niño que inventara sus propias palabras, después de trescientos y cuatrocien- tos no diría "quinientos", sino "cincocientos". Al permitir y fomentar exploraciónes lingüísticas de los niños, se vería que ellos tienen una etnolingüística mucho más fina que la de muchos ligüísticos profesionales. La prueba es que un niño puede aprender en un idioma nuevo en tres años sin diccionario sin intérprete, y ninguno de nosotros puede ya hacer otro tanto.

Si pasamos ahora de los lenguajes naturales a los lenguajes de la computación, llegaríamos a la necesidad de ubicar la informática en otro vértice del o c tógono. Ubiquémosla en el vértice inferior derecho. Me refiero a la informática en su sentido más general de ciencias de la información, de la codificación y decodificación, de los lenguajes formales, etc. Alguien podría decir que ello hace parte de la semiología general. Pero yo creo que la informática es mucho más compleja y rica que la mera codificación y decodificación, pues incluye todo el diseño, evaluación y manejo del "hardware" y el "software", etc. Puede estar cerca el día en que a través de las redes neurales se llegue directamente de la informática a la neurología.

Pero por el otro lado del octógono debemos ubicar las disciplinas clásicas que necesitaríamos para poder hacer investigaciones serias sobre las matemáticas como prácticas sociales 
de sujetos humanos, como serían la antropología, la sociología y la psicología. La dimensión diacrónica es también esencial para la compresión de esos fenómenos, y para ella es necesario ubicar también a la historia en un lugar preeminente del octógono. Empecemos pues por ella en el vértice superior izquierdo del octógono, cerca a la filosofía. En particular, no falta quien diga que una buena epistemología no es más que una buena historia de las ciencias, y en particular de las matemáticas. Pero yo creo que la episterı ılogía tiene una especificidad que desborda la historia, la que utiliza como una de sus fuentes, con el llamado por Piaget "método sociogenético", siendo los otros métodos el psicogenético y el formalizante.

En el vértice inferior izquierdo tendríamos la psicología, cerca de la neurología. Desafortunadamente, en un principio la psicología se absorbió casi toda la investigación en educación matemática, muchos de cuyos trabajos empezaron a aparecer en revistas de psicología. Todavía hay una fuerte tendencia a psicologizar la investigación en educación matemática, dejando un poco de lado al maestro con su saber pedagógico, a la microsociología de los grupos, y a otros aspectos socioculturales. Por eso es necesario agregar en el vértice central izquierdo una disciplina que recupere todos esos aspectos socio-culturales. Hubo una serie de estudios hechos por Michael Cole en el Africa sobre las matemáticas cotidianas de la tribu k'pelle de Liberia; más tarde se hicieron famosas las investigaciónes de Teresinha Carraher y Analucia
Schliemanm sobre las matemáticas extraescolares de los niños de la calle en el Brasil, los vendedores ambulantes, los loteros y "chanceros", etc.

En Colombia, Germán Mariño hizo investigaciones semejantes sobre los algoritmos utilizados por las, personas que se consideran analfabetas. La línea soviética de Vygotsky, Leontiev y El'konin destacó la importancia de los aspectos sociales, en particular del lenguaje de los adultos, en el aprendizaje de las matemáticas. Habría pues de poner en el vértice central izquierdo no sé si a la sociología o a la antropología, o a ambas, pues nunca he podido entender bien la diferencia ¿Será que la sociología no es sino la antropología de la tribu más cercana, o sea la propia, y la antropología la sociología de las tribus más lejanas? Escribamos pues en ese vértice "antropología cultural-sociología", para designar una disciplina de estudios de la cultura y la sociedad con cualquiera de los métodos apropiados para esos aspectos, que van desde el mas observacional no participantes, hasta la investigación-acción participativa más comprometida.

\section{EI OCTOGONO EN ACCION}

Para estudiar si el octógon o de disciplines que rodean a la investigación en educación matemática sí es productivo en la práctica de la misma, podemos comenzar haciéndonos una pregunta que impulse la conceptualización de la investigación en educación matemática: ¿Qué 
diferencia hay entre la manera como un psicólogo o un antropólogo mira a un niño que aprende a contar, o a sumar, restar, multiplicar, o dividir, y la manera como lo vería un maestro, - un investigador en educación matemática? ¿Están todos viendo lo mismo, o por lo menos están utilizando categorías que tengan una intersección no vacía que les permita comunicarse? upongamos que viene un psicólogo profesional muy versado en la psicología escolar y nos explica cómo aprende a dividir un niño según sus observaciones. El sociólogo le dirá que no tuvo en cuenta lo más importante, que es el ambiente escolar, !as interacciónes con los otros compañeros, las necesidades sociales que hacen de la división una herramienta útil, etc., y hará una descripción de un grupo de niños que están aprendiendo a dividir. EI maestro les dirá a ambos que sus niños no aprenden a dividir así como ellos dicen. ¿Qué puede decir el investigador en educación matemática que sea diferente de lo que digan los tres anteriores?

Tomemos un caso específico. Un profesor de la Universidad de Neiva que hace tutorias a maestros de primaria, me envió un algoritmo muy interesante de un niño que aprendió a dividir sin utilizar las multiplicaciones que los maestros enseñamos a hacer para dividir paso a paso con el algoritmo usual. El investigador en educación matemática mira ese algoritmo, no ya como un maestro; ni como un psicólogo, ni como un sociólogo. También empieza a buscar en la historia de las matemáticas qué otros algoritmos de división han ido apareciendo en distintas épocas y culturas. Investiga en el medio social del niño para ver si obtuvo ese algoritmo de fuentes extraescolares. Trata de modelar los procesos psicológicos que pudieron ocurrir en el cerebro del niño para producir esas inscripciones que aparecen en el papel en el que el niño consignó algunas divisiones. Analiza lingüísticamente las anotaciones que hace el mismo niño en ese papel, y las que hizo el maestro que detectó el algoritmo. Aquí escribe el niño: "Me pasé". Eso puede querer decir que el niño estaba duplicando el divisor repetidamente, hasta que se pasó, 0 sea hasta que llegó a un número más grande que el dividendo. El investigador empieza a generar.

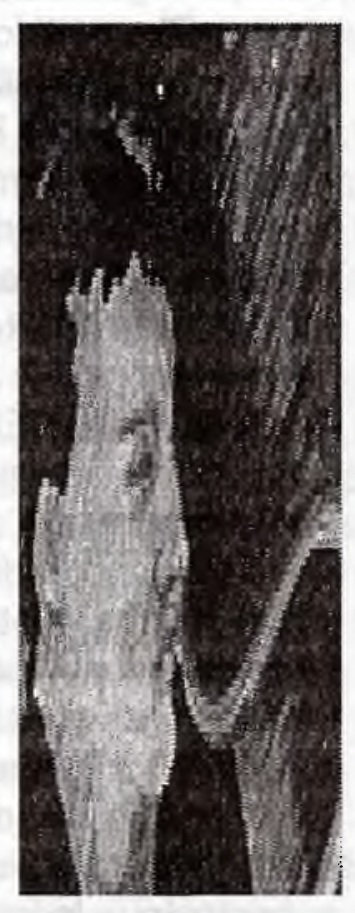
pos ibles procesos que podrían llevar a ese algoritmo; utiliza sus conocimientos de informática para ver si pueden programar un computador, o al menos hacer un diagrama de flujo, que calcule el cociente en la forma como el niño lo hizo; cae en la cuenta de la preferencia por la duplicación más bien que por la multiplicación que tienen los niños de ciertas edades, y compara con los algoritmos de multiplicación egipcios. De repente, las piezas del rompecabezas caen en sus puestos, y el cuadro del nuevo 
algoritmo aparece clararmente. Se diseña una pequeña investigación para ver si los niños de esa edad aprenden más facilmente ese algoritmo que el usual, y otra para ver cuál es la eficiencia del nuevo algoritmo, y los peligros de error que tiene con respecto al usual.

Vemos pues que el investigador en educación matemática sí utiliza a veces la visión del psicólogo, la del antropó-logo, la del historiador, la del especialista en informática, etc. Pero él es no especialisia en ninguna de esas disciplinas. Basta que tenga la información mínima suficiente para orientarse, y la curiosidad, la energía y la seriedad suficientes para aprender más y más de todo aquello que necesita para avanzar en su investigación. El investigador en educación matemática no necesita sacar ocho Ph.D.'s en las disciplinas del octógono, además de otro en matemáticas y otro en educación. Necesita si mantenerse informados en muchos frentes, $y$ tener las redes de amigos, colegas y fuentes de información adecuadas para mantenerse al día en aquellos aspectos que le interesan.

Por eso, en cierto sentido declarar que uno es investigador en educación matemática en un acto de soberbia intelectual inaudito, pues es pretender lo imposible, que es estár suficientemente informado en todos esos campos. Sin embargo, esto no debe desanimarnos para empezar por el sitio en donde estamos ubicados, como maestros, o sociólogos, o psicólogos, 0 investigadores educativos incipientes, y partir de la práctica de la investigación en educación matemática empezar a ubicarse dentro del proceso, a estudiar sus bases teóricas, a analizar las ventajas comparativas que uno tiene, a decidir por dónde empieza a estudiar algo de historia de las matemáticas, 0 de epistemología, etc. El maestro que haya estudiado la arimética y el álgebra del renacimiento, va a tener una visión muy distinta de sus estudios sobre la multiplicación y la división, o sobre el álgebra de bachillerato. EI psicólogo que estudie y practique la etnografía, y haya leído las investigaciones de Araceli de Tezanos sobre escuela y comunidad, va a entender aspectos antropológicos y didácticos que antes no podía ni siquiera percibir. El investigador y metodólogo que analiza esas mismas investigaciones etnográficas, y profundiza en los estudios que existen sobre el saber pedagógico de los maestros, y va entender que cuando los maestros se sonríen y se reconocen en esas investigaciones, se está dando allí un nuevo tipo de validación de ese tipo de investigación.

\section{LAS PREGUNTAS DE LA COMISION INTERNACIONAL DE INSTRUCCION MATEMATICA}

En el penúltimo congreso internacional de educación matemática de Budapest, en 1988, se conformó una comisión para profundizar en la conceptualización sobre sobre la investigación matemática, que presento su informe en el último congresó, celebrado en Quebec, Canadá, en agosto de 1992. Dicho informe fue publicado recientemente 
en el número de Diciembre de 1992 del "Bulletin of the International Commissión on Mathematics Instruction", y en forma resumida, en el número de Mayo de 1993 del "Journal for Research in Mathematics Education".

Por la oportunidad e importancia del tema, resumiré en esta última parte del artículo la preguntas que se hizo esta comisión internacional, con algunos comentarios personales al respecto.

\subsection{Preguntas sobre el punto de vista}

Podemos empezar por las preguntas que tiene más relación con la propuesta del

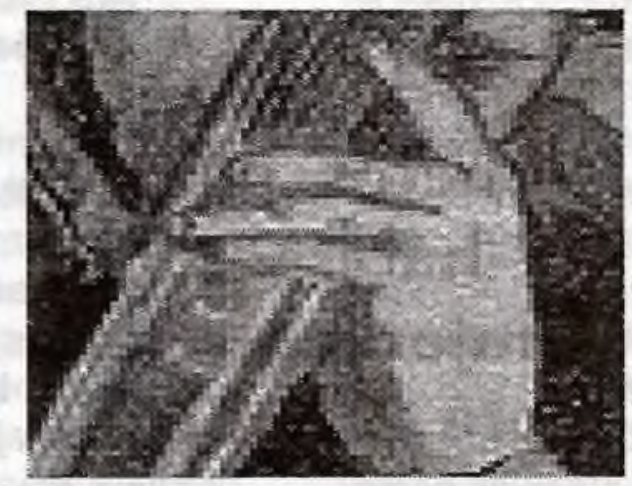

No basta pues el punto de vista interno; hay multitud de preguntas interesantes sobre la investigación matemática desde un punto de vista puramente externo. octógono de la investigación en educación matemática. ¿Desde dónde vamos a mirarla? ¿Desde un punto de vista más interno, como el de los dos practicantes de las misma, o desde un punto de vista más externo, como el de los dos observadores de la actividad de los practicantes? Desde este segundo punto de vista, un sociólogo podría observar cómo se ha ido institucionalizando la investigación en educación matemática; por ejemplo, vería cómo una red informal se transforma en la asociación para la investigación en la psicología de las matemáticas PMI, cómo aparecen los primeros boletines y las primeras revistas, cómo se van produciendo las primeras tesis doctorates en investigación en educación matemática,

\subsection{Preguntas sobre el objeto de la empresa}

Hay otro tipo de preguntas sobre el objeto de la empresa del investigador en educación matemática. Se ha propuesto que el objeto sea la enseñanza de las matemáticas. Pero surge entonces la discusión con aquellos que dicen que se trata más bien de investigar los procesos de aprendizaje de las matemáticas. En Medellín se acaba de inaugurar en la Universidad de Antioquia el archivo pedagógico nacional, en donde se están recogiendo los trabajos pedagógicos del siglo XIX, en la línea de investigación del grupo de Olga Lucia Zuluaga, Alberto Echeverri, 
Alberto Martínez, Stella Restrepo, Humberto Quiceno y sus colaboradores. Ellos han defendido el papel del maestro y la primacía de la enseñanza. Consideran que el aprendizaje es una noción psicologizante, que está deformando la misma identidad del maestro. Otras personas opinan que esa es una visión anticuada del maestro como transmisor de saberes a través de la palabra, cuando en realidad lo importante es saber qué es lo que está aprendiendo realmente el alumno, muchas veces en contra, $O$ al menos paralelamente a lo que le enseñan los maestros.

Están también los que se inclinan por poner como objeto de la investigación el binomio enseñanza-aprendizaje, que es considerado por algunos como un solo proceso, aunque otros distinguen alli dos procesos diferentes, y dirigen la atención hacia las relaciones entre esos dos procesos de enseñanza y de aprendizaje, y al contenido matemático involucrado en ellos. Otros piden incluír también la realidad del salón de clase, de la escuela, la comunidad y el sistema educativo formal, así como los canales de educación no formal y las concepciones socialmente compartidas acerca de las matemáticas y su enseñanza. Por ejemplo, James Stigler de la Universidad de Chicago ha encontrado que la apreciación de las mamás norteamericanas sobre el aprendizaje de las matemáticas es que algunos niños tienen una especie de don de Dios que hace que su cerebro esté hecho para aprender matemáticas, y que la mayoría no tiene ese don. En cambio, las mamás del Japón, Taiwan y Corea piensan que para aprender matemáticas basta un trabajo duro y constante, y que el que no las aprende es porque es perezoso. Esta apreciación tiene mucho efecto en el interés y el tiempo que invierten los alumnos orientales en aprender matemáticas, y los resultados de las comparaciones internacionales sobre rendimiento en matemáticas muestran enormes diferencias en favor de ellos.

\subsection{Preguntas sobre las metas y propósitos.}

Las pregunta sobre las metas y propósitos de la investigación en educación matemática se dirigen hacia las preferencias por las metas más pragmáticas o las más científicas. ¿Es lo más importante estudiar maneras de mejorar la enseñanza y de producir más eficaces aprendizajes, o es mejor concentrarse en la disciplina misma y en la acumulación de conocimiento serio y sistematizado, independientemente de sus posibles aplicaciones en el mundo escolar?

\subsection{Preguntas sobre la estructura del campo}

Otras preguntas versan sobre la estructura del campo intelectual de la educación matemática. ¿Debería estructurarse a lo largo de las disciplinas matemáticas como la aritmética, el álgebra, la geometría, el cálculo, etc.? ¿No sería mejor estructurar el campo según las teorias dominantes, como la piagetiana, la neo-piagetiana, la vygotskiana, la bruneriana, la del cambio conceptual 
de Ausubel, etc.? ¿Será más productivo estructurarla alrededor de lo que los franceses Ilaman "las problemáticas" ("problematiques"), 0 sea las constelaciones de preguntas, marco teóricos, conjeturas, resultados relacionados e indicaciones metodológicas? Algunos ejemplos de estas problemáticas podrían ser la de la interacción social en pequeños grupos; la del efecto de los métodos tutoriales individuales; las que se configuran alrededor del aprendizaje de conceptos especifícos como el fraccionario, o el de figura geométrica: la problemática de las demostraciones, etc.

Ese tipo de problemáticas van adquiriendo algunos de los rasgos de los paradigmas de Thomas Kuhn. Se dan preguntas compartidas, marcos teóricos comunes al grupo, métodos que se consideran como válidos, se van con conformando pequeñas comunidades sociológicas alrededor de cada problemática. etc.

Pero esto genera también preguntas alrededor de las problemáticas. ¿Cómo distinguir las problemáticas de la educación matemática de las de la psicología del aprendizaje, o de las de la didáctica de las matemáticas o de las de la epistemología de las matemáticas? Esta discusión nos devolvería al primer grupo de preguntas.

\subsection{Las preguntas por los resultados}

¿Qué se podría considerar como un resultado válido en investigación en educación matemática? Hay observaciones un poco intuitivas, pero de largo plazo y mucha perspicacia, como las que hizo Spinoza sobre la regla de tres hace ya más de tres siglos. Están también los niveles de los esposos van Hiele sobre el progreso en la comprensión de la geometría, que parecen puramente empíricos, pero han sido posteriormente objeto de muchas investigaciones y desarrollos curriculares. En cambio, hay otros resultados que si son producto de experimentos controlados. Pero aún estos últimos parecen no ser generalizables a las situaciones cotidianas de los maestros. ¿Se van a privilegiar los resultados empíricos de observaciones longitudinales, o se va a privilegiar la experimentación

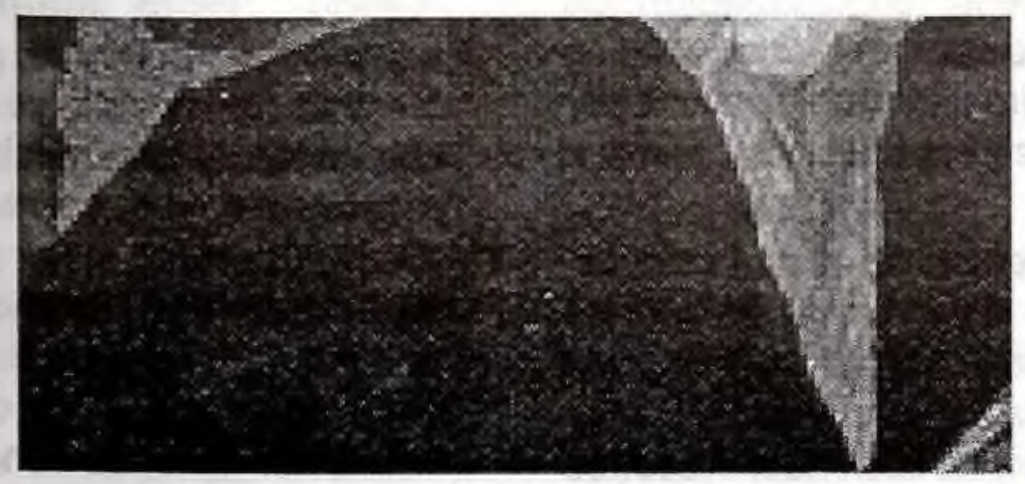


controlada? ¿Se van a preferir síntesis teóricas, o aplicaciones probadas en el salón de clase?

Para dar una indicación de los tipos de resultados que se han ido presentando en la literatura, y que tienen especial interés, propondré tres tipos de ellos:

4.5.1 Los economizadores de pensamiento. Cuando aparece una teoría, un método, una ley que condensa ciertas regularidades, este tipo de resultados permite economizar tiempo y esfuerzo de pensamiento, y permite pasar rápidamente a la práctica y al diseño de nuevas investigaciones.

4.5.2 Los energizadores de la práctica. No es fácil distinguir estos resultados de los anteriores, pues están muy relacionados con ellos. EI nombre de "energizadores" viene de esas pilas y baterías que aparecen ahora continuamente en la televisión. Ejemplos de los energizadores de la práctica de la educación matemática serían materiales, técnicas, juegos y sugerencias metodológicas que se derivan de la investigación, y que permitan a los maestros, a los autores y editores de textos, a los productores de materiales educativos, poner inmediatamente en circulación los resultados de esas investigaciones en una forma directamente utilizable por los maestros y los alumnos.

4.5.3 Los demoledores de ilusiones. Cada vez se observa más claramente que tanto los practicantes como los teóricos de la educación matemática tenemos muchas ilusiones. Nosotros mismos nos creemos, y hacemos creer a otros, muchos mitos y proyecciones sobre lo que es o lo que debería ser la educación matemática. Los demoledores de ilusiones son los resultados que derrumban esas creencias infundadas que todos damos por obvias. Por ejemplo, en la investigación sobre la calidad de la educación matemática recientemente presentada por el grupo SABER del Ministerio de Educación Nacional con el Instituto SER de Investigación y el ICFES, se encontró que mientras más televisión ven los niños, mejor les va en matemáticas. Sea cual sea la interpretación que se le de a esos resultados, y sean cuales fueren las preguntas ulteriores que ellos generan, esto derrumba muchas de las disculpas que teníamos los maestros para explicar por qué los niños de ahora no aprenden como los de antes.

\subsection{Las preguntas sobre la validez de los resultados}

Muy relacionados con las preguntas sobre los resultados son los últimos interrogantes que se planteó el comité internacional sobre lo que puede considerarse como suficiente para darle a un resultado la calificación de "válido". Estos interrogantes son especialmente díficiles, por involucrar las distinciones entre verdad, validez, confiabilidad, coherencia, objetividad relevancia y valor social de la investigación, generalizabilidad, replicabilidad, originalidad, impacto educativo y social de los resultados. etc., así como las operacionalizaciones de esos conceptos, y las salvaguardias metodológicas que 
permitan afirmar algunas de las anteriores calificaciones acerca de un resultado dado.

Esas son las preguntas permanentes para todo investigador, y en particular para todos los investigadores en educación matemática, ya vengan desde dentro de la práctica diaria del maestro, o desde la práctica del investigador universitario.

Así quedamos al final de este artículo con más preguntas que respuestas, pero ese es precisamente el atractivo magnético que ejerce la investigación en educación matemática sobre los que queremos practicarla.

[Este texto es una versión corregida por Teresa León Pereira y por el autor, de la grabación de una conferencia ofrecida por éste a la Red de Investigadores en Educación Matemática en la Biblioteca "Luis Angel Arango" de Santafé de Bogotá, el día 2 de septiembre de 1993] \&s

Tomado de la revista Enseñanza Universitaria.

Vol. 3 No. 2 - 1994 Universidad del Valle.

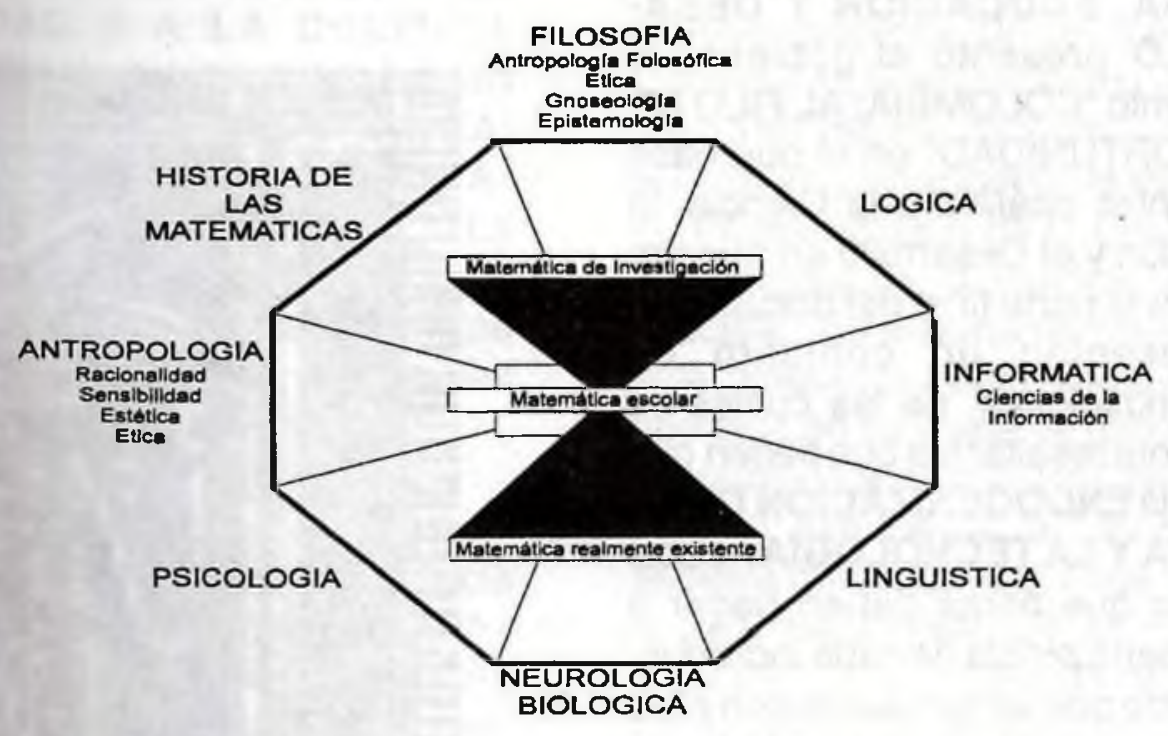

El octogono de la educación matemática 